PROCEEDINGS OF THE

AMERICAN MATHEMATICAL SOCIETY

Volume 127, Number 11, Pages 3367-3373

S 0002-9939(99)04869-8

Article electronically published on May 4, 1999

\title{
LARGE COMPONENTS OF PRINCIPAL SERIES AND CHARACTERISTIC CYCLES
}

\author{
JEN-TSEH CHANG
}

(Communicated by Roe W. Goodman)

\begin{abstract}
For a semisimple quasi-split real linear group which is also maximal in Kostant's sense, a theorem of Vogan asserts that there is a unique composition factor that is large in any principal series. We give a proof of this theorem using results of Schmid and Vilonen that establish a conjecture of Barbasch and Vogan about characteristic cycles. As a byproduct, we obtain some information about the characteristic cycles of the localized $K$-equivariant sheaves of these principal series.
\end{abstract}

\section{INTRODUCTION}

1.1. Suppose $G_{\mathbb{R}}$ is a real semisimple quasi-split matrix group that lies, of finite index, in a connected complex algebraic group defined over $\mathbb{R}$. We use $\mathfrak{g}_{\mathbb{R}}$ to denote the Lie algebra of $G_{\mathbb{R}}$ and $\mathfrak{g}$ for its complexification; a similar convention will be used for other groups as well.

Suppose moreover $G_{\mathbb{R}}$ is maximal in the sense of [5], i.e., $\operatorname{Ad} G_{\mathbb{R}}$ contains all the elements in the adjoint group $\operatorname{Int}(\mathfrak{g})$ which normalize $\mathfrak{g}_{\mathbb{R}}$. Then a deep result of Vogan [11] asserts that, for each principal series of $G_{\mathbb{R}}$, there exists a unique irreducible subquotient component that is large - it is called the Vogan-Speh component of the principal series. By definition, an irreducible representation is large if its annihilator in the enveloping algebra of $\mathfrak{g}$ is the minimal primitive ideal; equivalently, according to a result of Joseph (see [11]), if its Gelfand-Kirillov dimension is maximal. Vogan's result was one important ingredient in Kostant's proof [5] that an irreducible representation which is large has a Whittaker model; the converse was shown also by Kostant earlier. In this note we give a different proof of Vogan's theorem using results of Schmid and Vilonen [8], [9], which constitute a proof of a conjecture of Barbasch and Vogan concerning characteristic cycles of representations. Our intent has been to pursue possible applications of this remarkable conjecture. Indeed the proof presented here is rather simple modulo the deep results of Schmid and Vilonen. As a byproduct of this, we obtain some information about the characteristic cycle of the localized $K$-equivariant sheaves of these principal series (see 1.3 below).

Received by the editors January 22, 1997 and, in revised form, January 21, 1998.

1991 Mathematics Subject Classification. Primary 22E46.

We thank J. Cogdell for bringing us into this particular subject, and the referee for helpful suggestions. This research was supported in part by an Arts and Sciences Summer Research grant in 1996 from the Oklahoma State University.

(C)1999 American Mathematical Society 
1.2. We now give a sketch of the idea. Fix a maximal compact subgroup $K_{\mathbb{R}}$ of $G_{\mathbb{R}}$ once and for all; we use the following notation convention: removing the subscript $\mathbb{R}$ amounts to taking complexification. Suppose $M$ is an admissible representation of $G_{\mathbb{R}}$. The characteristic cycle of $M$, denoted by $\mathcal{C} h(M)$, is an algebraic cycle of nilpotent $K$-orbits in $(\mathfrak{g} / \mathfrak{k})^{*}$ (the linear dual of $\mathfrak{g} / \mathfrak{k}$ ) defined using the underlying algebraic $(\mathfrak{g}, K)$-structure of $M$. The Gelfand-Kirillov dimension of $M$ is simply the dimension of the support of $\mathcal{C} h(M)$. The characteristic cycle contains a lot more information than just the Gelfand-Kirillov dimension from a structure view-point. The Barbasch-Vogan conjecture says that the characteristic cycle can by computed by the asymptotics of the local distribution character of $M$ near the origin (of $\mathfrak{g}_{\mathbb{R}}$ ) (cf. [1]). More specifically, the Fourier transform of the local distribution character of $M$ admits an asymptotic expansion near the origin, whose leading term turns out to be a linear combination of Liouville measures on nilpotent $G_{\mathbb{R}}$-orbits in $\sqrt{-1} \mathfrak{g}_{\mathbb{R}}^{*}$. Forgetting the canonical Liouville measure, this determines a linear combination of nilpotent $G_{\mathbb{R}}$-orbits, which we will refer to as the asymptotic cycle of $M$ (it's called the Barbasch-Vogan invariant in [8]), and denote by $\mathcal{A} s(M)$. The conjecture asserts that the Kostant-Sekiguchi correspondence - a canonical bijection between nilpotent $G_{\mathbb{R}^{-}}$orbits in $\sqrt{-1} \mathfrak{g}_{\mathbb{R}}^{*}$ and nilpotent $K$-orbits in $(\mathfrak{g} / \mathfrak{k})^{*}$ - exchanges $\mathcal{A s}(M)$ and $\mathcal{C} h(M)$ (see 2.2.1(1) and 2.4.1). We remark that both operations $\mathcal{C} h$ and $\mathcal{A} s$ are additive from the semigroup of the admissible representations of $G_{\mathbb{R}}$ with equal Gelfand-Kirillov dimensions.

1.3. Let $X$ be the flag variety of the Lie algebra $\mathfrak{g}$ and $T^{*} X$ its cotangent space. The Rossmann formula for characters, established in general cases by Schmid and Vilonen [8], calculates the asymptotic cycle in terms of the homology of $T^{*} X$. Let $\mu$ be the moment map from $T^{*} X$ to $\mathcal{N}^{*}$, the nilpotent variety in $\mathfrak{g}^{*}$ (defined to be the set corresponding to $\mathcal{N}$, the variety of all nilpotent elements in $\mathfrak{g}$ via the identification through the Killing form). Using the localization theory and the Matsuki correspondence (or other means, see [8]), the representation $M$ assigns a $G_{\mathbb{R}^{-}}$equivariant complex of sheaves on $X$ (more accurately an object in the $G_{\mathbb{R}^{-}}$ equivariant derived category of $X$ ) which we denote by $\mathcal{M}$. Taking the characteristic cycle of $\mathcal{M}$ according to Kashiwara and Schapira [2], we get a Lagrangian cycle, denoted by $C C(\mathcal{M})$, on $T^{*} X$. The formula in [8] asserts that $\mathcal{A} s(M)$ can be computed by integrating a suitable (canonical) cohomology class (arising from the infinitesimal character of $M$ ) over the fiber of the moment map $\mu$ in $C C(\mathcal{M})$. In general, the objects $\mathcal{M}, C C(\mathcal{M})$, and the integration are very difficult to compute. However in our situation, $G_{\mathbb{R}}$ being quasi-split and $M$ being a principal series representation, all these objects are quite easy to compute - mainly because $\mathcal{M}$ is supported on the closed $G_{\mathbb{R}}$-orbit; in fact all the multiplicities in the asymptotic cycle are given by one. This formal procedure can also be considered in the setting of the $K$-action in exactly the same way and $\mathcal{C} h(M)$ can be obtained directly by pushing down through the moment map of suitable characteristic cycle of the localization of $M$. However in this setting, the localization of $M$ is supported on the full flag variety (being the closure of the open $K$-orbit); its characteristic cycle is much more intractable than that in the $G_{\mathbb{R}}$-setting. This is precisely the reason why we need to go to the $G_{\mathbb{R}}$-action side in order to prove this result. As an application of our method, we determine the "major" part of the characteristic cycle of the localized $K$-equivariant sheaf of $M$ (see 2.5). 


\section{THE PROOF}

2.1. As in 1.1, we retain the assumption on $G_{\mathbb{R}}$ but will not pose the condition of being maximal unless stated otherwise. Let $\theta$ be the Cartan involution corresponding to the choice of the maximal compact subgroup $K_{\mathbb{R}}$; we have $\mathfrak{g}_{\mathbb{R}}=\mathfrak{k}_{\mathbb{R}}+\mathfrak{p}_{\mathbb{R}}$, with $\mathfrak{p}_{\mathbb{R}}$ the(-1)-eigenspace of $\theta$. Fix a $\theta$-stable maximally split Cartan subgroup $H_{\mathbb{R}}=T_{\mathbb{R}} A_{\mathbb{R}}$ of $G_{\mathbb{R}}$ with $T_{\mathbb{R}} \subset K_{\mathbb{R}}$ and $A_{\mathbb{R}} \subset \exp \mathfrak{p}_{\mathbb{R}}$. We choose an Iwasawa decomposition $G_{\mathbb{R}}=K_{\mathbb{R}} A_{\mathbb{R}} N_{\mathbb{R}}$. Since $G_{\mathbb{R}}$ is quasi-split, $T_{\mathbb{R}}$ is the centralizer of $A_{\mathbb{R}}$ in $K_{\mathbb{R}}$, and $\mathfrak{b}:=\mathfrak{h}+\mathfrak{n}$ is a Borel subalgebra of $\mathfrak{g}$. Write $B_{\mathbb{R}}$ for $T_{\mathbb{R}} A_{\mathbb{R}} N_{\mathbb{R}}$. We now state the result we shall prove using the method outlined in the introduction.

2.1.1. Theorem (Vogan, Corollary 6.7 of [11]). For any character $\delta$ of $T_{\mathbb{R}}$ and $\nu$ of $A_{\mathbb{R}}$, the principal series representation $M=\operatorname{In} d_{B_{\mathbb{R}}}^{G_{\mathbb{R}}}(\delta \otimes \nu \otimes i d)$ has a large composition factor $Y$, which appears with multiplicity one. $Y$ is unique up to conjugation by outer automorphisms of $G_{\mathbb{R}}$ coming from Int $(\mathfrak{g})$, and fixing the principal series.

There are various equivalent statements for a representation to be large (see [5] for details). In the proof given in [11], it is relatively easy to show the existence of such a composition factor, however the statement concerning multiplicity one and the uniqueness is much more involved. We shall see that the characteristic cycle consideration sees all these at the same time.

2.2. We now collect certain facts concerning nilpotent orbits, following [6]. Let $F$ be the set of all $a \in A=\exp \mathfrak{a}$, considered as a subgroup in $\operatorname{Int}(\mathfrak{g})$, such that $a^{2}$ is the identity; $F$ is a finite abelian group of order $2^{r}$ if $r$ is the $\mathbb{R}$-dimension of $A_{\mathbb{R}}$. Let $\mathcal{N}_{\mathfrak{k}}^{*}:=\mathcal{N}^{*} \cap(\mathfrak{g} / \mathfrak{k})^{*}$ and $\mathcal{N}_{\mathbb{R}}^{*}:=\mathcal{N}^{*} \cap\left(\mathfrak{g} / \mathfrak{g}_{\mathbb{R}}\right)^{*}$ be relevant subspaces in the nilpotent cone $\mathcal{N}^{*}$. Note that $\mathcal{N}_{\mathbb{R}}^{*}$ is simply the purely imaginary part of $\mathcal{N}^{*}$ since $G_{\mathbb{R}}$ is quasi-split.

2.2.1. Theorem (Kostant-Rallis and Sekiguchi). (1) There is a canonical one-toone correspondence between the $K$-orbits in $\mathcal{N}_{\mathfrak{k}}^{*}$ and the $G_{\mathbb{R}}$-orbits in $\mathcal{N}_{\mathbb{R}}^{*}$, and the corresponding orbits have the same $\mathbb{R}$-dimension.

(2) $K$ and $G_{\mathbb{R}}$ act on $\mathcal{N}_{\mathfrak{k}}^{*}$ and $\mathcal{N}_{\mathbb{R}}^{*}$ respectively with finitely many orbits.

(3) $F \cdot A d(K)$ acts on $\mathcal{N}_{\mathfrak{k}}^{*}$ with exactly one open dense orbit.

(4) $A d\left(G_{\mathbb{R}}\right) \cdot F$ is the set of all $a \in \operatorname{Int}(\mathfrak{g})$ such that $a \cdot \mathfrak{g}_{\mathbb{R}}=\mathfrak{g}_{\mathbb{R}}$.

(5) The open $K$-orbits in $\mathcal{N}_{\mathfrak{k}}^{*}$ and the open $G_{\mathbb{R}}$-orbits in $\mathcal{N}_{\mathbb{R}}^{*}$ are contained in the open $\operatorname{Int}(\mathfrak{g})$-orbit in $\mathcal{N}^{*}$.

The first statement is the Kostant-Sekiguchi correspondence [10], (2) and (4) are Theorem 2 and Propositions 1, 2 of Chapter 1 in [6], and (3) is Theorem 6 of Introduction in [6]. The last statement is also standard; for lack of a direct reference, we give an argument here. Suppose $e$ is an arbitrary element in an open $K$-orbit in $\mathcal{N}_{\mathfrak{k}}^{*}$ and $\{x, e, f\}$ a normal S-triple containing $e$. Then $x$ is conjugated, via $\operatorname{Int}(\mathfrak{g})$, to $x^{\prime}:=e+f \in \mathfrak{p}$ and $\operatorname{dim} \mathfrak{g}^{x^{\prime}}=\operatorname{dim} \mathfrak{a}+\operatorname{dim} \mathfrak{t}$, which is the rank of $\mathfrak{g}$ (by Theorem 4 and Proposition 9 in Chapter 1 of [6]). By Theorem 5.2 of [4], it follows that this $\mathrm{S}$-triple is also principal in $\mathcal{N}^{*}$, i.e., $e$ lies in the open $\operatorname{Int}(\mathfrak{g})$-orbit in $\mathcal{N}^{*}$. In light of $(1)$, the same holds for open $G_{\mathbb{R}}$-orbits in $\mathcal{N}_{\mathbb{R}}^{*}$ by counting the dimension; thus (5) follows. Note that the number of open orbits in either case is given by the cardinality of $F / F \cap \operatorname{Ad}(K)=F / F \cap \operatorname{Ad}\left(G_{\mathbb{R}}\right)=F / F \cap \operatorname{Ad}\left(K_{\mathbb{R}}\right)$.

Since $F \subset \exp (\mathfrak{a})$, any element in $F$ will leave the principal series in Theorem 2.1.1 fixed. Now if $G_{\mathbb{R}}$ is maximal, i.e., $\operatorname{Ad}\left(G_{\mathbb{R}}\right) \supset F$, then by the above two 
theorems, we see that any principal series has exactly one large composition factor; this is the result mentioned in 1.1.

2.3. We proceed to the computation of the asymptotic cycle of the principal series following [8]. First of all, let $\lambda=d(\delta \otimes \nu) \in \mathfrak{h}^{*}$; then $\chi_{\lambda}$ (in Harish-Chandra's notation) is the infinitesimal character of $M=\operatorname{Ind}_{B_{\mathbb{R}}}^{G_{\mathbb{R}}}(\delta \otimes \nu \otimes \mathrm{id})$. In [8], a surjective homomorphism $\beta$ is defined from the category of $G_{\mathbb{R}}$-equivariant derived category (with twist $-\lambda$ ) to the category of virtual $G_{\mathbb{R}}$ representations of infinitesimal character $\chi_{\lambda}$; specifically - though we shall make no use of this - by (2.3) of [8], it is given by

$$
\mathcal{F} \mapsto \sum(-1)^{p} \operatorname{Ext}^{p}\left(D \mathcal{F}, \mathcal{O}_{X}(\lambda)\right),
$$

where $D$ is the Verdier dual.

To describe an object mapped to $M$ under $\beta$, let $S$ be the $G_{\mathbb{R} \text {-orbit of the Borel }}$ subalgebra $\mathfrak{b}$ in $X$, the flag variety of $\mathfrak{g}$. Note that $S$ is the unique closed $G_{\mathbb{R}}$-orbit in $X$; we denote by $i$ the inclusion. Now $S \simeq G_{\mathbb{R}} / B_{\mathbb{R}}$; write $\tau$ for the unique $G_{\mathbb{R}^{-}}$ equivariant sheaf of locally constant sections on $S$ induced by the character which is dual to $\delta \otimes\left(\nu \otimes e^{\rho}\right) \otimes$ id on $B_{\mathbb{R}}$, where $\rho \in \mathfrak{h}^{*}$ is the half of the trace on $\mathfrak{g} / \mathfrak{b}$. It is then well-known (see for example the introduction of [3]) that

$$
\beta\left(i_{*}(\tau)\right)=M
$$

we shall see that the exact form of the character giving rise to $\tau$ is not so important for our purpose. By Theorem 6.2 of [8], the asymptotic cycle of $M$ is then given by

$$
\left(\operatorname{gr} \mu_{*}\right)_{\lambda} \circ C C\left(i_{*}(\tau)\right) \text {. }
$$

Recall that $C C$ stands for taking characteristic cycle and $\mu$ is the moment map. The operation $\left(\operatorname{gr} \mu_{*}\right)_{\lambda}$ is the push-down functor defined in (5.2) of [8] - it amounts

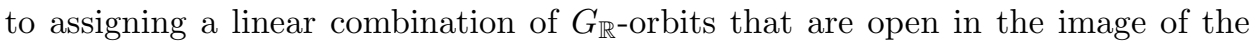
support of $C C\left(i_{*}(\tau)\right)$ under $\mu$, where the coefficient of such an orbit is obtained by integrating a canonical cohomology class (determined by $\lambda$ ) over its generic fiber in the cycle $C C\left(i_{*}(\tau)\right)$. We note that the component in degree zero of this canonical cohomology class is "1" - this is crucial to our argument in 2.4.1.

2.3.3. Lemma. (1) $C C\left(i_{*}(\tau)\right)=T_{S}^{*} X$, the conormal bundle of $S$ in $X$.

(2) The image of $T_{S}^{*} X$ under the moment map $\mu$ is the union of closures of all the open $G_{\mathbb{R}}$-orbits in $\mathcal{N}_{\mathbb{R}}^{*}$; moreover, over the open orbits, $\mu$ is one-to-one.

Proof. Since $\tau$, being induced from a character, is locally isomorphic to the constant sheaf on $S$ and $S$ is a closed submanifold of $X$, the first statement is standard and follows from (9.4.8) of [2]. Recall that $\mu: T^{*} X \rightarrow \mathcal{N}^{*}$ is one-to-one over the open dense $\operatorname{Int}(\mathfrak{g})$-orbit in $\mathcal{N}^{*}$; in fact it is a resolution of singularity of $\mathcal{N}^{*}$. The statement (2) follows from Theorem 2.2.1(5) and the fact that every regular element, i.e., those in the open nilpotent $\operatorname{Int}(\mathfrak{g})$-orbit in $\mathfrak{g}_{\mathbb{R}}$, is conjugated via $G_{\mathbb{R}}$ to an element in $\mathfrak{n}_{\mathbb{R}}$ - the fiber of $T_{S}^{*} X$ over the base point representing the Borel subalgebra $\mathfrak{b}$. This fact is also well-known; for lack of a direct reference we give an argument here. Suppose $e$ is an element in an arbitrary open $G_{\mathbb{R}}$-orbit in $\mathcal{N}_{\mathbb{R}}^{*}$; by Lemma 1.4 of [10], we may assume, up to a conjugation by $G_{\mathbb{R}}$, that $e$ lies in a strictly normal S-triple $\{x, e, f\}$. In particular $x \in \mathfrak{p}_{\mathbb{R}}$ and is a regular element in $\mathfrak{g}$ (cf. the argument for 2.2.1 (5)). Therefore up to a conjugation by $K_{\mathbb{R}} \subset G_{\mathbb{R}}$, we may assume that $x$ lies in the positive Weyl chamber of $\mathfrak{a}_{\mathbb{R}}$ determined by roots in $\mathfrak{n}_{\mathbb{R}}$. Thus $e \in \mathfrak{n}_{\mathbb{R}}$ since $[x, e]=2 e$. This completes the proof of the lemma. 
2.4. Since $\mu$ is one-to-one over the open $G_{\mathbb{R}^{-} \text {-orbits by the above lemma, there is }}$ nothing to do to compute $\left(\operatorname{gr} \mu_{*}\right)_{\lambda} \circ C C\left(i_{*}(\tau)\right)$. In light of Lemma 2.3.3, this gives the first statement of the following

2.4.1. Proposition. (1) $\mathcal{A} s(M)=\sum O$, where the sum runs over all open $G_{\mathbb{R}^{-}}$ orbits $O$ in $\mathcal{N}_{\mathbb{R}}^{*}$; in particular each orbit appears with multiplicity one.

(2) $\mathcal{C} h(M)=\sum \overline{\mathcal{O}}$, where the sum runs over all open $K$-orbits $\mathcal{O}$ in $\mathcal{N}_{\mathfrak{k}}^{*}$; in particular each irreducible component appears with multiplicity one.

(3) The Gelfand-Kirillov dimension of $M$ is given by $\operatorname{dim} \mathfrak{n}$.

In light of 2.2.1(1), the Barbasch-Vogan conjecture says that statements (1) and (2) are equivalent. Noticing that open $K$-orbits are of maximal dimension in $\mathcal{N}_{\mathfrak{k}}^{*}$, (3) follows.

2.4.2. We can now prove the main Theorem 2.1.1. By the above proposition, there exists an irreducible composition factor say $Y$ in the principal series representation $M$ whose characteristic cycle is given by a sum of certain open $K$-orbits in $\mathcal{N}_{\mathfrak{k}}^{*}$, moreover it appears with multiplicity one. Any element $a \in F$ fixes the principal series and thus $a \cdot Y$ would be a composition factor of the principal series with characteristic cycle given by $a \cdot \mathcal{C} h(Y)$, which is either equal to $\mathcal{C} h(Y)$ or else shares no common components with $\mathcal{C} h(Y)$ again due to the multiplicity one property. Taking into account Theorem 2.2.1(4), the theorem follows.

2.5. We close this article with a remark on the characteristic cycle of the localized $K$-equivariant sheaf on $X$ corresponding to the principal series $M$. Recall the notations in 2.2 .

2.5.1. Definition. A $K$-orbit $Z$ in $X$ is called small if, under the moment map, its conormal bundle $T_{Z}^{*} X$ is birational to its image in $\mathcal{N}_{\mathfrak{k}}^{*}$.

2.5.2. Lemma. Recall that the group $G_{\mathbb{R}}$ is quasi-split.

(1) Small $K$-orbits exist and are closed in $X$.

(2) $F \cdot A d K$ acts transitively on the union of all small $K$-orbits; in particular, the number of small $K$-orbits in $X$ is given by the cardinality of $F /(F \cap A d K)$.

(3) The union of all conormal bundles of small K-orbits is birational to $\mathcal{N}_{\mathfrak{k}}^{*}$ under the moment map.

Proof. By 2.2.1 (5), the open $K$-orbits in $\mathcal{N}_{\mathfrak{k}}^{*}$ consist of all the principal nilpotent elements in $\mathcal{N}_{\mathfrak{k}}^{*}$; therefore the moment map $\mu$ is birational from $\bigcup_{Z} T_{Z}^{*} X$ (union over all $K$-orbits $Z$ ) to $\mathcal{N}_{\mathfrak{k}}^{*}$. This says that small $K$-orbits exist and also gives (3). Let $Z$ be an arbitrary $K$-orbit in $X$; then we can pick a point say $z$ in $Z$ whose corresponding Borel subalgebra $\mathfrak{b}_{z}$ contains a Cartan subalgebra $\mathfrak{h}_{z}$ which is the complexification of a $\theta$-stable Cartan subalgebra $\mathfrak{h}_{\mathbb{R}}$ of $\mathfrak{g}_{\mathbb{R}}$ (Corollary to Theorem 1 in [7]). Therefore we have the usual notion of (compact and noncompact) imaginary roots, complex roots and real roots for the root system $\Phi\left(\mathfrak{g}, \mathfrak{h}_{z}\right)$. Write $\mathfrak{b}_{z}=\mathfrak{h}_{z}+\mathfrak{n}_{z}$ and let $\Delta_{z}^{+}$be the set of simple roots for the positive root system so that $\mathfrak{n}_{z}=$ $\sum_{\alpha>0} \mathfrak{g}_{\alpha}$. Identifying $\mathfrak{g}^{*}$ with $\mathfrak{g}$ via the Killing form, we may consider the fiber

$$
\left(T_{Z}^{*} X\right)_{z}=\left(\mathfrak{g} /\left(\mathfrak{k}+\mathfrak{b}_{z}\right)\right)^{*} \subset \mathfrak{g}^{*}
$$


as a subspace of $\mathfrak{g}$. Now

$$
\begin{aligned}
\mathfrak{k}+\mathfrak{b}_{z}=\mathfrak{h}_{z} & +\sum_{\alpha>0 \text { imaginary }} \mathfrak{g}_{\alpha}+\sum_{\alpha>0 \text { compact }} \mathfrak{g}_{-\alpha}+\sum_{\alpha>0 \text { complex }} \mathfrak{g}_{\alpha} \\
& +\sum_{\alpha>0 \text { complex and } \theta \alpha>0, A_{-\alpha} \in \mathfrak{g}_{-\alpha}}\left(A_{-\alpha}+\theta A_{-\alpha}\right) \\
& +\sum_{\alpha>0 \text { complex and } \theta \alpha<0} \mathfrak{g}_{\theta \alpha}+\sum_{\alpha>0 \text { real }}\left(\mathfrak{g}_{\alpha}+\mathfrak{g}_{-\alpha}\right) .
\end{aligned}
$$

The last two terms arise as a consequence of the fact that $A_{\alpha}+\theta\left(A_{\alpha}\right) \in \mathfrak{k}$ for any root vectors $A_{\alpha}$. Therefore,

$$
\mathfrak{g} /\left(\mathfrak{k}+\mathfrak{b}_{z}\right) \simeq \sum_{\alpha>0} \mathfrak{g}_{\text {noncompact }}+\sum_{\alpha>0 \text { complex and } \theta \alpha>0, A_{-\alpha} \in \mathfrak{g}_{-\alpha}}\left(A_{-\alpha}-\theta A_{-\alpha}\right) .
$$

Under the above identification, the fiber $\left(T_{Z}^{*} X\right)_{z}$ is given by

$$
\sum_{\alpha>0 \text { noncompact }} \mathfrak{g}_{\alpha}+\sum_{\alpha>0 \text { complex and } \theta \alpha>0, A_{\alpha} \in \mathfrak{g}_{\alpha}}\left(A_{\alpha}-\theta A_{\alpha}\right) \subset \mathcal{N}_{\mathfrak{k}}^{*} .
$$

If the $K$-orbit $Z$ is not closed in $X$, then $\mathfrak{b}_{z}$, hence $\Delta_{z}^{+}$is not $\theta$-stable (cf. Proposition 2 in $\S 3$ of [7]). Thus there exists $\alpha \in \Delta_{z}^{+}$so that either $\alpha$ is real or $\alpha$ is complex and $\theta \alpha<0$. By a theorem of Kostant (Theorem 5.3 in [4]), an element $x \in \mathfrak{n}_{z}$ is principal if and only if $\left(x, \mathfrak{g}_{-\beta}\right) \neq 0$ for any simple $\operatorname{root} \beta$ in $\Delta_{z}^{+}$; here $($,$) is the$ Killing form. Therefore, when $Z$ is not closed, no elements in the fiber (2.5.3) are principal and $Z$ is not small. Thus any small $K$-orbits must be closed. This gives (1). Since $F \cdot \operatorname{Ad} K$ acts with only one open dense orbit in $\mathcal{N}_{\mathfrak{k}}^{*}$ (see 2.2.1(3)), it must act transitively on the union of small $K$-orbits. This gives (2) and completes the proof of the lemma.

2.5.4. Corollary. (1) Retaining the notations above, a closed $K$-orbit $Z$ is small if and only if the set of simple roots $\Delta_{z}^{+}$contains no compact imaginary roots.

(2) The conormal bundle of each (closed) small $K$-orbit in $X$ appears exactly once in the characteristic cycle of the localized $K$-equivariant sheaf corresponding to the principal series $M$.

Proof. (1) is a consequence of the above proof. For (2), note that the characteristic cycle considered in the corollary pushes down to the characteristic cycle $\mathcal{C} h(M)$ in the same fashion described in 2.3 (cf. (2.5.6) in [1] or Theorem 6.1 in [8]). The above lemma says that, under the moment map, the pre-image of the closure of each open $K$-orbit in $\mathcal{N}_{\mathfrak{k}}^{*}$ is precisely the conormal bundle of some small $K$-orbit in $X$. The lemma then follows from 2.4.1(2).

2.5.5. Corollary 2.5.4(1) says that any quasi-split group has a $\theta$-stable positive root system on a fundamental Cartan subalgebra with no simple compact imaginary roots. In general, most $\theta$-stable positive root systems on a fundamental Cartan subalgebra do not have this property. One can also define the notion of small $K$-orbits for groups that are not quasi-split, however such orbits generally do not exist. 


\section{REFERENCES}

1. J.-T. Chang, Asymptotics and characteristic cycles for representations of complex groups, Compo. Math. 88 (1993), 265-283. MR 94i:22031

2. M. Kashiwara and P. Schapira, Sheaves on Manifolds, Springer-Verlag, 1990. MR 92a:58132

3. M. Kashiwara and W. Schmid, Equivariant derived category and representations of semisimple Lie groups, preprint, 1994.

4. B. Kostant, The principal three dimensional subgroup and the Betti numbers of a complex simple Lie group, Amer. J. Math. 81 (1959), 973-1032. MR 22:5693

5. B. Kostant, On Whittaker vectors and representation theory, Invent. Math. 48 (1978), 101184. MR 80b:22020

6. B. Kostant and S. Rallis, Orbits and representations associated with symmetric spaces, Amer. J. Math. 93 (1971), 753-809. MR 47:399

7. T. Matsuki, Orbits on affine symmetric spaces under the action of parabolic subgroups, Hiroshima Math. J. 12 (1982), 307-320. MR 83k:53072

8. W. Schmid and K. Vilonen, Characters, characteristic cycles, and nilpotent orbits, Geometry, Topology and Physics for Raoul Bott, International Press, 1995, pp. 329-340. MR 96g:22021

9. W. Schmid and K. Vilonen, Characteristic cycles of constructible sheaves, Invent. Math. 124 (1996), 451-502. MR 96k:32016

10. J. Sekiguchi, Remarks on nilpotent orbits of a symmetric pair, J. Math. Soc. Japan 39 (1987), 127-138. MR 88g:53053

11. D. Vogan Jr., Gelfand-Kirillov dimension for Harish-Chandra modules, Invent. Math. 48 (1978), 75-98. MR 58:22205

Department of Mathematics, Oklahoma State University, Stillwater, Oklahoma 74078-0613

E-mail address: changj@math.okstate.edu 